\title{
Associations between Interleukin-32 Gene Polymorphisms rs12934561 and rs28372698 and Susceptibilities to Bladder Cancer and the Prognosis in Chinese Han Population
}

\author{
Jie Yang, ${ }^{1,2}$ Zhongyu Jian, ${ }^{1}$ Pengfei Shen, ${ }^{1}$ Yunjin Bai, ${ }^{1}$ Yin Tang, ${ }^{1}$ and Jia Wang $\mathbb{D}^{1}$ \\ ${ }^{1}$ Department of Urology, Institute of Urology, West China Hospital, Sichuan University, Chengdu, Sichuan 610041, China \\ ${ }^{2}$ Department of Urology, Chengdu First People's Hospital, Chengdu, Sichuan 610041, China \\ Correspondence should be addressed to Jia Wang; wangjiawch@163.com \\ Jie Yang and Zhongyu Jian contributed equally to this work.
}

Received 20 April 2020; Revised 20 July 2020; Accepted 24 October 2020; Published 5 November 2020

Academic Editor: Hubertus Himmerich

Copyright (C) 2020 Jie Yang et al. This is an open access article distributed under the Creative Commons Attribution License, which permits unrestricted use, distribution, and reproduction in any medium, provided the original work is properly cited.

\begin{abstract}
The proinflammatory chemokine interleukin-32 is related to various diseases, including cancer. However, it has never been associated with bladder cancer (BC). To detect whether there is a relationship between the $I L-32$ gene polymorphisms (rs12934561 C/T and rs28372698 T/A) and BC, the study enrolled 170 non-muscle-invasive bladder cancer (NMIBC) patients, 151 muscle-invasive bladder cancer (MIBC) patients, and 437 healthy controls. The polymerase chain reaction-restriction fragment length polymorphism (PCR-RFLP) method was used for the IL-32 single-nucleotide polymorphism (SNP) genotyping. Statistical analysis was performed using SNPstats online analysis software and SPSS software. Our data revealed that the CC homozygous genotype of rs12934561 in BC patients was significantly higher than that in controls $(P=0.03, \mathrm{OR}=1.47,95 \% \mathrm{CI}$ $=1.04-2.08)$, and the percentage of TC genotype carriers was relatively less than that of controls $(P=0.001, \mathrm{OR}=0.61,95 \% \mathrm{CI}$ $=0.45-0.82)$. Furthermore, the TT homozygous genotype of rs28372698 was associated with a significantly lower overall survival rate in $\mathrm{MIBC}$ patients $(P=0.028, \mathrm{OR}=2.77,95 \% \mathrm{CI}=1.11-6.90)$. The $I L-32$ gene polymorphism rs12934561 might be associated with increased BC risk, and the rs 28372698 might participate in the prognosis of BC patients. Therefore, they could be potential forecasting factors for the prognosis of MIBC patients.
\end{abstract}

\section{Introduction}

Bladder cancer (BC) is the tenth most common cancer according to the International Agency for Research on Cancer (IARC), with 549,393 new cases worldwide in 2018 (1). Seventy-five percent of the total burden occurs in men, and $60 \%$ of the incidence rate and $50 \%$ of the mortality rate occur in the less developed regions of the world. In 2018, 82,270 new cases and 38,208 deaths were recorded in China, which revealed an estimated increase of 30,000 cases and 20,000 deaths compared with the data of 2012 (1). According to these reports, only about $20 \%$ of BC patients have muscleinvasive bladder cancer (MIBC), which is responsible for most of the cancer-specific deaths. The remaining $80 \%$ of the patients present with non-muscle-invasive bladder cancer (NMIBC) (1-3).

Common $\mathrm{BC}$ risk factors include tobacco smoking and exposure to industrial paints, petroleum products, and other chemical carcinogens (2-4). However, in recent years, increasing evidence has demonstrated a genetic predisposition towards it (5). Furthermore, the first-degree relatives of $\mathrm{BC}$ patients have a twofold higher risk of developing BC, showing that genetic factors play a crucial role in the initiation and progression of this disease.

Interleukin-32, a proinflammatory cytokine, was first detected as the product of natural killer cell transcript 4 (NK4) in 1992 (6) and was officially renamed as IL-32 by Kim et al. in 2005 (7). Its encoding gene $I L-32$ is located on 
TABLE 1: Characteristics of the study population.

\begin{tabular}{|c|c|c|c|}
\hline Characteristics & NMIBC group & MIBC group & Controls \\
\hline Sample size & 170 & 151 & 437 \\
\hline \multicolumn{4}{|l|}{ Sex } \\
\hline Male & $131(77.1 \%)$ & $122(80.8 \%)$ & $336(76.9 \%)$ \\
\hline Female & $39(22.9 \%)$ & $29(19.2 \%)$ & $101(23.1 \%)$ \\
\hline Age at first diagnosis (mean \pm SD) & $62.14 \pm 12.87$ & $65.70 \pm 11.06$ & $63.86 \pm 6.94$ \\
\hline \multicolumn{4}{|l|}{ Smoking status } \\
\hline Smokers & $85(50.0 \%)$ & $82(54.3 \%)$ & $199(45.5 \%)$ \\
\hline Nonsmokers & $85(50.0 \%)$ & $69(45.7 \%)$ & $238(54.5 \%)$ \\
\hline \multicolumn{4}{|l|}{ Clinical stage } \\
\hline $\mathrm{Ta}$ & $10(5.9 \%)$ & - & - \\
\hline $\mathrm{T} 1$ & $160(94.1 \%)$ & - & - \\
\hline $\mathrm{T} 2$ & - & 89 (58.9\%) & - \\
\hline $\mathrm{T} 3 \mathrm{a}$ & - & $34(22.5 \%)$ & - \\
\hline $\mathrm{T} 3 \mathrm{~b}$ & - & $17(11.3 \%)$ & - \\
\hline $\mathrm{T} 4$ & - & $11(7.3 \%)$ & - \\
\hline \multicolumn{4}{|l|}{ Tumor grade } \\
\hline Low grade & $114(67.1 \%)$ & $23(15.2 \%)$ & - \\
\hline High grade & $56(32.9 \%)$ & $128(84.8 \%)$ & - \\
\hline
\end{tabular}

TABle 2: Primer sequences for genotyping two SNPs in the IL-32 gene.

\begin{tabular}{|c|c|c|c|}
\hline SNP ID & Primer sequence & Restriction enzyme & Allele (bp) \\
\hline \multirow[t]{2}{*}{ rs12934561 } & F: 5'-GGCCTCACTCCTCACACAGT-3' & Hpy188III & $C(20+155)$ \\
\hline & R: 5' -CCCACAGGTGTTGGTTTCC-3' & & $\mathrm{T}(175)$ \\
\hline \multirow[t]{2}{*}{ rs28372698 } & F: $5^{\prime}$-GTCAGAAGGACCTGGTCAGC-3' & Hpy188III & A (115) \\
\hline & R: 5'-GTTGGAGGGGTGGCTAGTC-3' & & $\mathrm{T}(21+94)$ \\
\hline
\end{tabular}

the human chromosome $16 \mathrm{p} 13.3$, is approximately $1,200 \mathrm{bp}$ full-length, and consists of eight exons (6). IL-32 is mainly produced by activated T cells, NK cells, epithelial cells, and blood monocytes (7), and it has nine splice variants IL-32 $\alpha$, IL-32 $\beta$, IL-32 $\gamma$, IL-32 $\delta$, IL-32 $\varepsilon$, IL-32 $\theta$, IL-32 $\zeta$, IL-32 $\eta$, and IL-32small (IL-32sm) $(8,9)$. IL-32 has been implicated in many inflammatory diseases and cancers, including rheumatoid arthritis (10), chronic obstructive pulmonary disease (COPD) (11), lymphoma (12), head and neck squamous cell carcinoma (HNSCC) (13), thyroid cancer (TC) (14), hepatocellular carcinoma (HCC) (15), lung cancer (LC) (16-18), esophageal cancer (19), gastric cancer (GC) $(20,21)$, pancreatic cancer (22), colorectal cancer $(\mathrm{CRC})(23,24)$, renal cell carcinoma (RCC) $(25)$, breast cancer (26), and endometrial cancer (EC) (27).

Recently, several reports have clearly indicated that two single nucleotide polymorphisms (SNPs) in the IL-32 gene sequence (rs12934561 and rs28372698) were associated with cancer susceptibility (LC, GC, TC, EC, and CRC) $(14,16,24$, 27-29). However, no relationship has been established between IL-32 and BC. Therefore, we selected these two SNPs (rs12934561 and rs28372698) of IL-32 to determine their differences in BC patients and healthy controls in the Chinese Han population.

\section{Material}

2.1. Participants' Clinical Characteristics. A case-control study which enrolled 321 unrelated BC individuals (mean \pm SD: $63.82 \pm 12.17$ years (NMIBC group: $62.14 \pm 12.87$ years; MIBC group: $65.70 \pm 11.06$ years)) and 437 healthy controls (mean \pm SD: $63.86 \pm 6.94$ years) was approved by the hospital ethics committee, and informed consent was provided by all the participants. The subjects were from the West China Hospital of Sichuan University between 2007 and 2012. All participants with personal or family history of BC or other severe diseases such as other types of cancers, or those who had undergone radiotherapy or chemotherapy, were excluded from the study. Patients' clinical and follow-up data were collected every 6 months for 5 years by telephone calls. All tumor tissues resected from BC patients were confirmed by histopathological analysis, and the clinical characteristics are summarized in Table 1. All of the participants were genetically unrelated individuals of the Han population living in the Sichuan province of China.

2.2. Genotyping. As shown in Table 2, polymerase chain reaction (PCR) primers of the two SNPs were designed using 
TABLE 3: Distribution of SNPs in $I L-32$ among patients and controls and their association with bladder cancer risk.

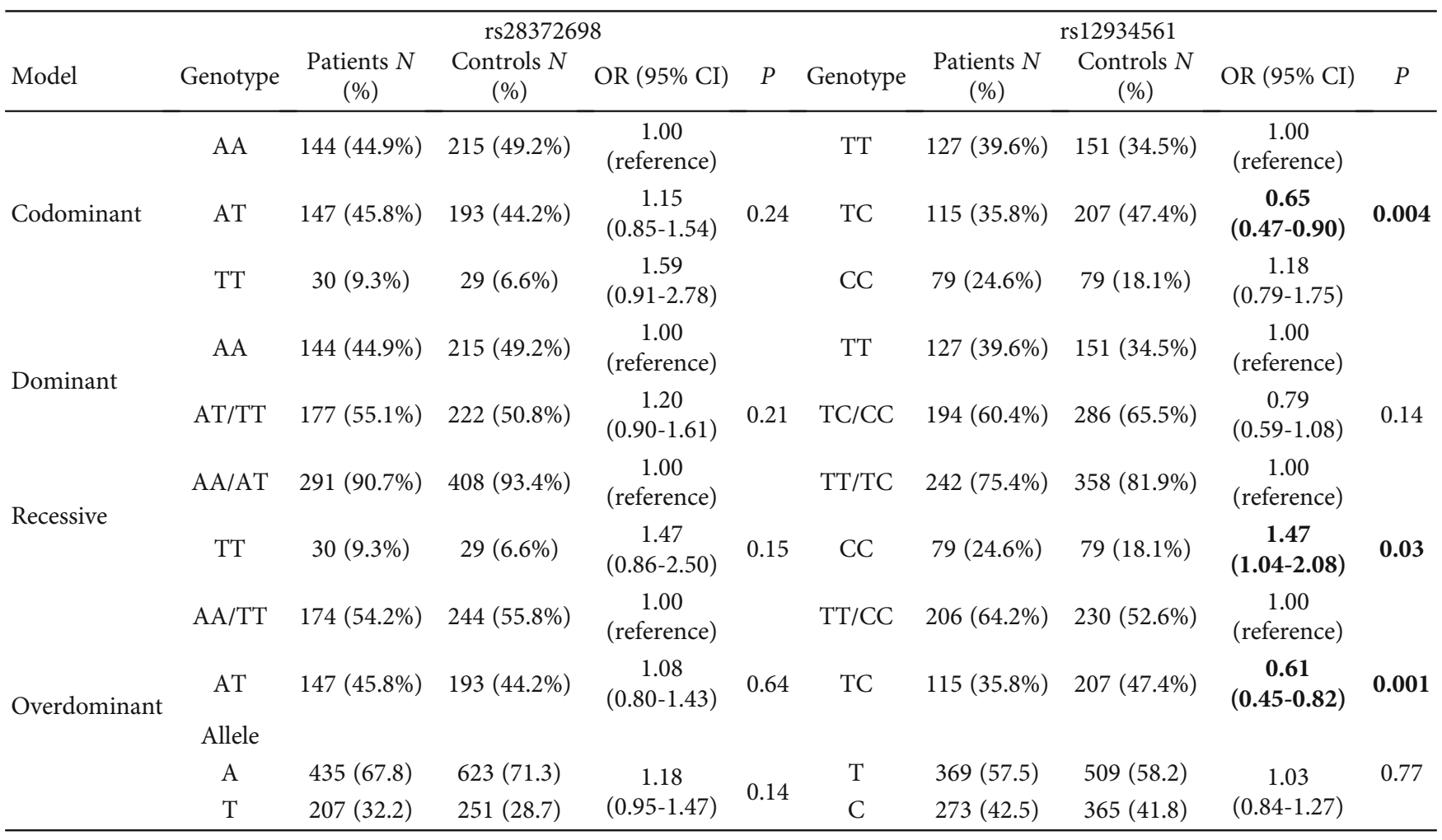

$N$ corresponds to the number of individuals. Boldfaced values indicate a significant difference at the $5 \%$ level.

Primer 3 web version 4.1.0. (http://primer3.ut.ee/) (30). The genetic DNA of each individual was extracted from a $200 \mu \mathrm{L}$ EDTA-anticoagulated peripheral blood sample using a DNA isolation kit from BioTeke (Peking, China). Genotyping was performed using PCR-restriction fragment length polymorphism (PCR-RFLP). The DNA fragments that contained the polymorphisms were amplified in a volume of $10 \mu \mathrm{L}$, including $100 \mathrm{ng}$ extracted genomic DNA, 2.7 picomole primers of each SNP, and $5 \mu \mathrm{L} 2 \mathrm{x}$ power Taq PCR Master Mix (BioTeke, Peking, China). The PCR annealing temperature was $60^{\circ} \mathrm{C}$ for $30 \mathrm{~s}$. After PCR termination, the products were digested by a restriction enzyme, as shown in Table 2, and the digested fragments were separated on a $6 \%$ polyacrylamide gel and stained with $1.5 \mathrm{~g} / \mathrm{L}$ of argent nitrate. Finally, DNA sequencing analysis was used to confirm the genotypes, and approximately $10 \%$ of the randomly selected samples were $100 \%$ in agreement with the results after performing the repeated assays.

2.3. Statistical Analysis. The SNPstats online analysis software was used to evaluate the genotypic association, including the codominant, dominant, recessive, and overdominant genetic models (31), and the Hardy-Weinberg equilibrium was calculated using the chi-squared test. The effects of different genotypes and alleles were evaluated by odds ratio (OR) and respective 95\% confidence intervals (95\% CI). Kaplan-Meier univariate analysis plots and Cox regression multivariate survival analysis model were used to estimate the relationships of $I L-32$ genotypes with patient outcomes. The level of significance was set at $P<0.05$.

\section{Results}

3.1. Susceptibility between the IL-32 Genotypes and BC. The genotype distributions of these two SNPs follow the HardyWeinberg equilibrium $(P>0.05)$ in our groups. The effects of $I L-32$ genotypes and allele frequencies on BC patients are presented in Table 3. As shown, for rs12934561, the homozygous genotype (CC) in the recessive genetic model was significantly higher in BC patients than that in controls $(24.6 \%$ vs. $18.1 \%, P=0.03, \mathrm{OR}=1.47,95 \% \mathrm{CI}=1.04-2.08$ ), indicating an increased risk for BC susceptibility. Compared with the TT/CC genotypes, the TC genotype was associated with a lower risk for $\mathrm{BC}$ in the overdominant model $(P=0.001$, OR $=0.61,95 \% \mathrm{CI}=0.45-0.82)$. No significant differences were observed between BC susceptibility and the rs 28372698 genotype or allele distribution.

3.2. Clinical Characteristics. To gain further insights into the relationship between these two SNPs of $I L-32$ and BC, patients with different genotypes were stratified by mean age ( $\leq 64$ and $>64$ years old), sex (male and female), smoking status (smokers and nonsmokers), tumor grade (low-grade and high-grade), and tumor stage (Ta-T1 and T2-T4) (Supplementary Table 1). However, no significant relationship was detected for any subgroup of the SNPs after adjusting for common risk factors $(P>0.05)$.

3.3. The Effects of IL-32 SNP Genotypes on Patient Outcome. During the follow-up, all of the involved BC patients were tracked every six months. At the end of our study, 50 patients 


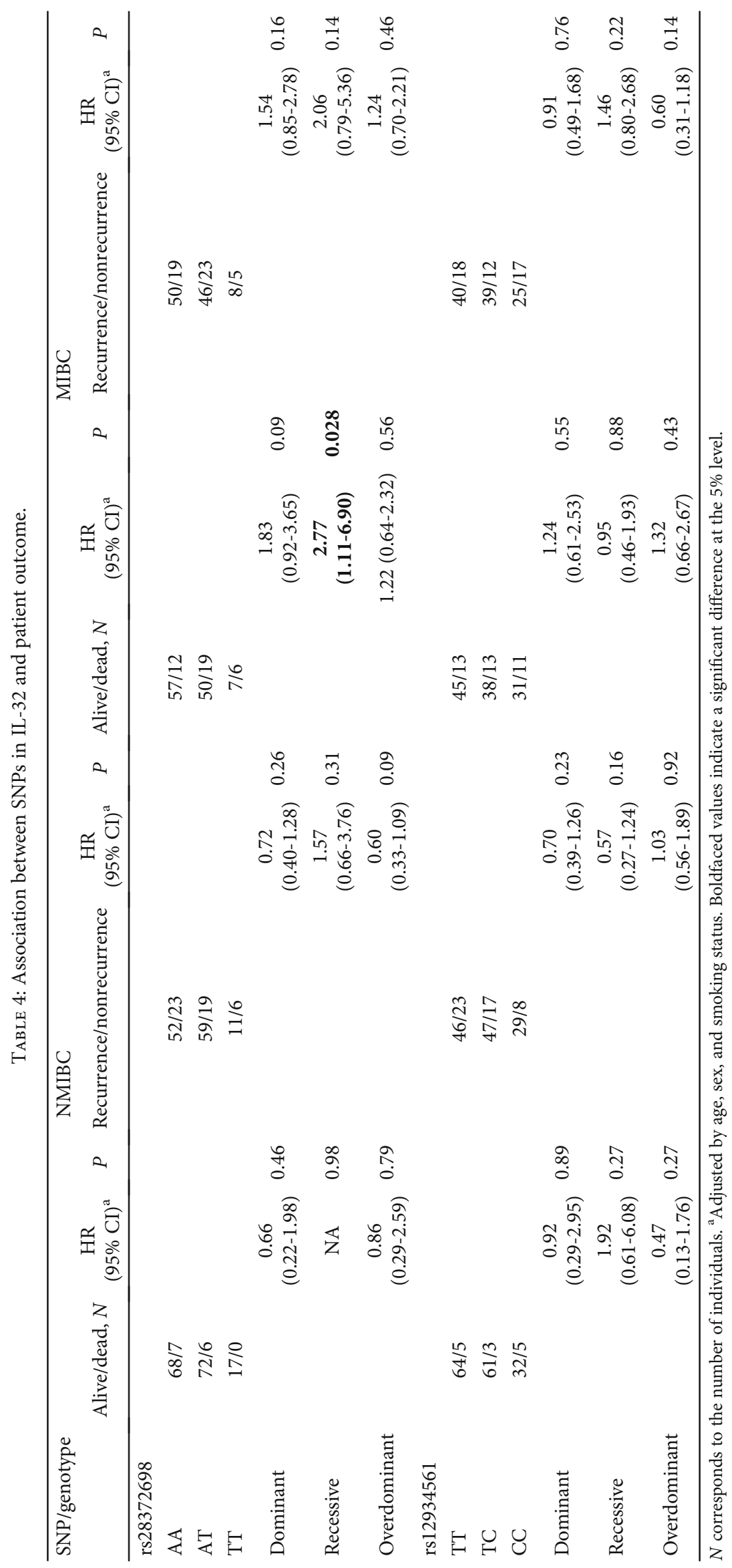




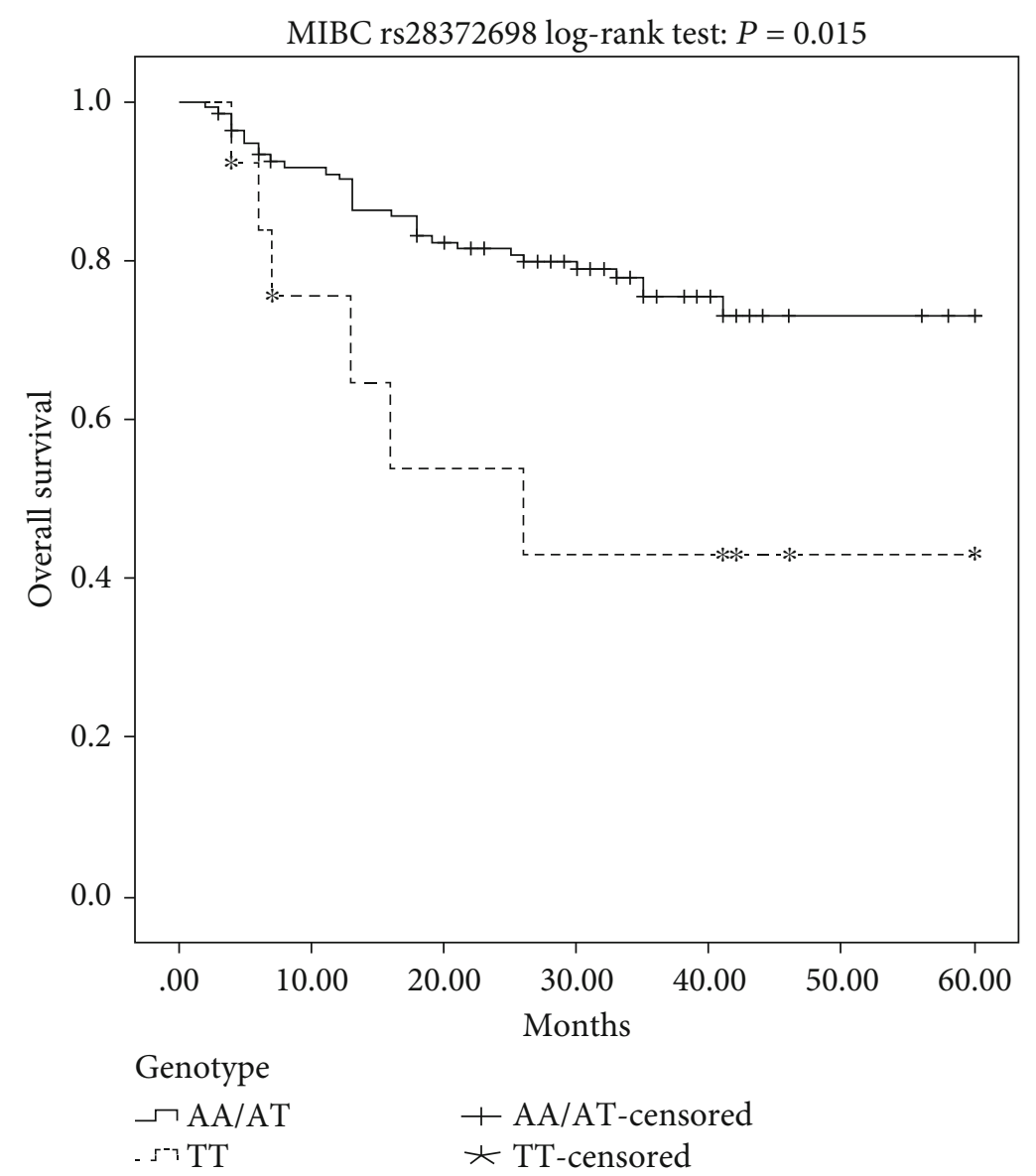

Figure 1: Kaplan-Meier overall survival curves for all of the analyzed MIBC patients categorized by IL-32 rs 28372698 in the recessive genetic models.

(15.6\%, NMIBC: 13 cases, MIBC: 37 cases) died of BC and 95 patients (29.6\%, NMIBC: 48 cases, MIBC: 47 cases) relapsed. Following the stratification of patients by tumor stage (MIBC and NMIBC), we conducted Kaplan-Meier survival analyses and multivariate Cox survival analyses; the associations between SNPs of $I L-32$ and BC patient outcomes are summarized in Table 4.

Kaplan-Meier plots indicated a significantly worse prognosis of MIBC patients carrying the TT homozygous genotype of $I L-32$ rs 28372698 compared to that of AA or AT genotypes (log-rank test: $P=0.015$, Figure $1 ; P=0.025$, Figure 2). Furthermore, as shown in Table 4, the multivariate survival analyses reiterated that the TT genotype carriers $(P=0.028$, OR $(95 \% \mathrm{CI})=2.77(1.11-6.90))$ had a worse overall survival rate in MIBC patients after adjustment for age, sex, and smoking status. However, no significant relationship was detected between the overall survival rate and another SNP (rs12934561) or between these two SNPs and the recurrencefree survival rate.

\section{Discussion}

Proinflammatory cytokines, such as tumor necrosis factor $\alpha$ (TNF- $\alpha$ ), interleukin-8 (IL-8), interleukin-6 (IL-6), and interleukin- $1 \beta$ (IL-1 $\beta$ ), could be induced by IL-32, which is often associated with inflammatory and oncogenic diseases
(7, 32-34). However, no homologous relationship has been found between the structural basis of IL-32 and the known cytokines, and no extracellular signaling receptor of IL-32 1has been detected until now $(7,35,36)$. IL-32 has nine splice variants, and all of the isoforms present differences in secondary structures, which lead to the variant tertiary protein structure and protein function (37).

Substantial reports have shown that IL-32 has different roles in various situations and pathways. Mabilleau and Sabokbar reported that IL-32 is capable of inducing a strong activation of ERK1/2 and Akt signaling and stimulating the release of interleukin-4 (IL-4) and interferon- $\gamma$ (IFN- $\gamma$ ) in osteoclast formation and activation (38). The transcriptional coactivator p300 (EP300) and death-associated protein kinase-1 (DAPK-1) were found to occupy the inflammatory network nodes of IL-32, which affect both TNF-receptor 1dependent and TNF-receptor 1-independent pathways (39). Yousif et al. demonstrated that IL-32 is associated with NF$\kappa \mathrm{B}$ and p38 MAPK pathways in esophageal tumors in vivo (19), whereas in vitro, Oh et al. found that it is involved in the NF- $\kappa$ B-STAT3 signaling pathway in colon cancer cells (40). Park et al. suggested that IL-32 $\beta$ could increase the invasion and migration of breast cancer through the EGFRSTAT3 pathway (26).

Several studies on the SNP of $I L-32$ in cancer have been reported in recent years, and $I L-32$ has even been linked to 


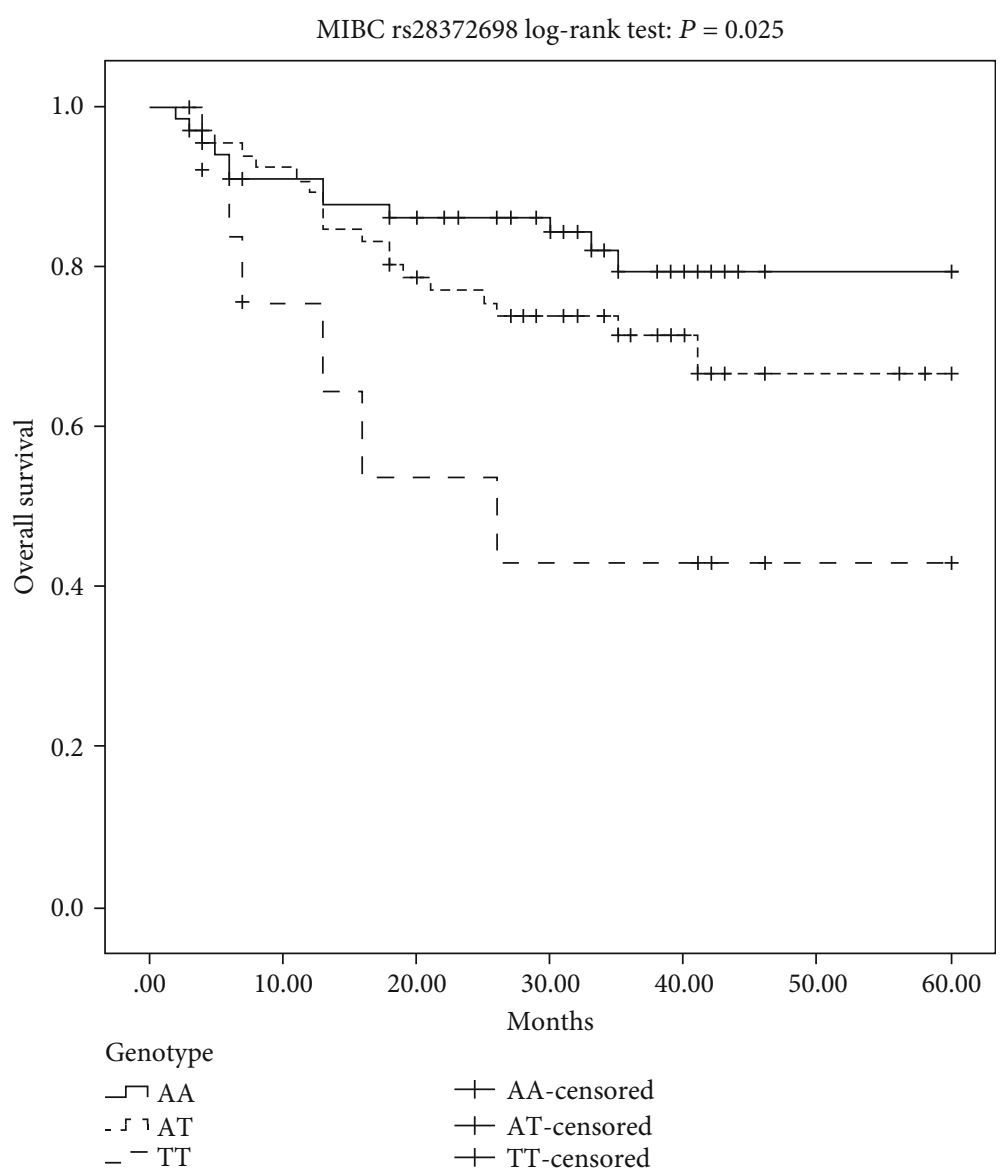

FIGURE 2: Kaplan-Meier overall survival curves for all of the analyzed MIBC patients categorized by $I L-32$ rs 28372698 in the codominant genetic models.

the patient outcome in some cancers. Our data revealed that the CC genotype of rs12934561 in $I L-32$ was associated with an increased risk of $\mathrm{BC}$, which is consistent with findings of previous studies in which the CC genotype was shown to relate closely with an increased susceptibility in lung cancer and endometrial cancer $(16,27)$. Moreover, Wang et al. showed that lung squamous carcinoma patients with the TT genotype of rs12934561 present a relatively poor survival rate compared with that of other patients (16). However, in our study, we demonstrated that the TC heterozygotes of rs12934561 are associated with a decreased risk of BC, which might be caused by the variant effects and functional pathways of nine isoforms in different organizations.

The rs28372698 T/A genetic variants were located on the $5^{\prime}$-UTR in the promoter region of $I L-32$. Plantinga et al. investigated a cohort of 139 TC patients and 138 healthy controls who carried the rs28372698 T/A genetic variants, revealing an increased risk of TC in patients with genetic variants of IL-32. Those patients required higher doses of cumulative radioactive iodine (RAI) to achieve successful tumor remission (14). Gonzalez-Hormazabal et al. used a combined attribute network implemented in multifactor dimensionality reduction software to analyze the gene-gene interactions between $I L-8-251 \mathrm{~A}>\mathrm{T}$ and $I L-32$ rs28372698 T/A, and their results showed that the homozygote for both $I L-8-251 \mathrm{~T}$ and
IL-32 rs28372698 $\mathrm{T}$ alleles presents a 2.63 -fold risk in the developing gastric cancer (29). Furthermore, in moderate and well-differentiated lung cancer, the $\mathrm{T}$ allele of rs28372698 is associated with a poor prognosis (16), which is consistent with our data. In our study, the TT genotype of rs28372698 in $I L-32$ was associated with a lower overall survival rate of MIBC patients, which indicated that IL-32 might be a potential biomarker for the prognosis of $\mathrm{BC}$.

\section{Conclusions}

In conclusion, to the best of our knowledge, this is the first study to demonstrate the relationship between IL-32 and BC. The results indicate that SNP rs12934561 may be a potential risk factor for BC processes, and SNP rs28372698 is a significant forecast factor for BC prognosis. Nevertheless, our study has some limitations in terms of sample size and in the absence of the expression level of IL-32 in participants. The types and frequencies of genetic polymorphisms in variant ethnic populations differ, whereas only a cohort of southwest China was genotyped in this study. Thus, further studies in different populations and with larger sample sizes are required to reveal the potential function and mechanism of IL-32 in BC and to confirm these findings. 


\section{Data Availability}

The data used to support the findings of this study are currently under embargo while the research findings are commercialized. Requests for data, 6 months after publication of this article, will be considered by the corresponding authors.

\section{Conflicts of Interest}

The authors declare that they have no conflict of interest.

\section{Authors' Contributions}

Jie Yang and Zhongyu Jian contributed equally to this work.

\section{Supplementary Materials}

Supplementary Table 1: association between SNPs in $I L-32$ and patient's characteristics. (Supplementary Materials)

\section{References}

[1] R. L. Siegel, K. D. Miller, and A. Jemal, "Cancer statistics, 2015," CA: A Cancer Journal for Clinicians, vol. 65, no. 1, pp. 5-29.

[2] M. Babjuk, A. Böhle, M. Burger et al., "EAU Guidelines on Non-Muscle-invasive Urothelial Carcinoma of the Bladder: Update 2016," European Urology, vol. 71, no. 3, pp. 447-461.

[3] M. Burger, J. W. F. Catto, G. Dalbagni et al., "Epidemiology and risk factors of urothelial bladder cancer," European Urology, vol. 63, no. 2, pp. 234-241.

[4] N. D. Freedman, D. T. Silverman, A. R. Hollenbeck, A. Schatzkin, and C. C. Abnet, "Association between smoking and risk of bladder cancer among men and women," JAMA, vol. 306 , no. 7 , pp. 737-745.

[5] D. Volanis, T. Kadiyska, A. Galanis, D. Delakas, S. Logotheti, and V. Zoumpourlis, "Environmental factors and genetic susceptibility promote urinary bladder cancer," Toxicology Letters, vol. 193, no. 2, pp. 131-137.

[6] C. A. Dahl, R. P. Schall, H. L. He, and J. S. Cairns, "Identification of a novel gene expressed in activated natural killer cells and T cells," Journal of Immunology, vol. 148, pp. 597-603, 1992.

[7] S.-H. Kim, S.-Y. Han, T. Azam, D.-Y. Yoon, and C. A. Dinarello, "Interleukin-32: A Cytokine and Inducer of TNF $\alpha$," Immunity, vol. 22, no. 1, pp. 131-142.

[8] D. H. Sohn, T. T. Nguyen, S. Kim et al., "Structural characteristics of seven IL-32 variants," Immune Network, vol. 19, no. 2, article e8, 2019.

[9] Y. J. E. Sloot, J. W. Smit, L. A. B. Joosten, and R. T. NeteaMaier, "Insights into the role of IL-32 in cancer," Seminars in Immunology, vol. 38, pp. 24-32.

[10] L. A. B. Joosten, M. G. Netea, S.-H. Kim et al., "IL-32, a proinflammatory cytokine in rheumatoid arthritis," Proceedings of the National Academy of Sciences, vol. 103, no. 9, pp. 32983303.

[11] F. Calabrese, S. Baraldo, E. Bazzan et al., "IL-32, a novel proinflammatory cytokine in chronic obstructive pulmonary disease," American Journal of Respiratory and Critical Care Medicine, vol. 178, no. 9, pp. 894-901, 2008.
[12] H. Suga, M. Sugaya, T. Miyagaki et al., "The role of IL-32 in cutaneous T-cell lymphoma," Journal of Investigative Dermatology, vol. 134, no. 5, pp. 1428-1435.

[13] S. Guenin, M. Mouallif, P. Hubert et al., "Interleukin-32 expression is associated with a poorer prognosis in head and neck squamous cell carcinoma," Molecular Carcinogenesis, vol. 53, no. 8, pp. 667-673.

[14] T. S. Plantinga, I. Costantini, B. Heinhuis et al., "A promoter polymorphism in human interleukin-32 modulates its expression and influences the risk and the outcome of epithelial cellderived thyroid carcinoma," Carcinogenesis, vol. 34, no. 7, pp. 1529-1535, 2013.

[15] Y. H. Kang, M.-Y. Park, D.-Y. Yoon et al., "Dysregulation of overexpressed IL-32 $\alpha$ in hepatocellular carcinoma suppresses cell growth and induces apoptosis through inactivation of NF- $\kappa$ B and Bcl-2," Cancer Letters, vol. 318, no. 2, pp. 226-233.

[16] Y. Wang, Y. Yang, Y. Zhu, L. Li, F. Chen, and L. Zhang, "Polymorphisms and expression of IL-32: impact on genetic susceptibility and clinical outcome of lung cancer," Biomarkers, vol. 22, no. 2, pp. 165-170, 2017.

[17] C. Sorrentino and E. Di Carlo, "Expression of IL-32 in human lung cancer is related to the histotype and metastatic phenotype," American Journal of Respiratory and Critical Care Medicine, vol. 180, no. 8, pp. 769-779.

[18] Q. Zeng, S. Li, Y. Zhou et al., "Interleukin-32 contributes to invasion and metastasis of primary lung adenocarcinoma via NF-kappaB induced matrix metalloproteinases 2 and 9 expression," Cytokine, vol. 65, no. 1, pp. 24-32.

[19] N. G. Yousif, F. G. Al-amran, N. Hadi, J. Lee, and J. Adrienne, "Expression of IL-32 modulates NF- $\kappa \mathrm{B}$ and p38 MAP kinase pathways in human esophageal cancer," Cytokine, vol. 61, no. 1, pp. 223-227.

[20] M. Pavlovic, N. Gajovic, M. Jurisevic et al., "Diverse expression of IL-32 in diffuse and intestinal types of gastric cancer," Gastroenterology Research and Practice, vol. 2018, Article ID 6578273, 9 pages, 2018.

[21] S. Ishigami, T. Arigami, Y. Uchikado et al., "IL-32 expression is an independent prognostic marker for gastric cancer," Medical Oncology, vol. 30, no. 2.

[22] A. Nishida, A. Andoh, O. Inatomi, and Y. Fujiyama, "Interleukin-32 expression in the pancreas," The Journal of Biological Chemistry, vol. 284, no. 26, pp. 17868-17876, 2009.

[23] Y. Yang, Z. Wang, Y. Zhou, X. Wang, J. Xiang, and Z. Chen, "Dysregulation of over-expressed IL-32 in colorectal cancer induces metastasis," World Journal of Surgical Oncology, vol. 13 , no. 1 .

[24] L. Shamoun, B. Kolodziej, R. E. Andersson, and J. Dimberg, "Protein expression and genetic variation of IL32 and association with colorectal cancer in Swedish patients," Anticancer Research, vol. 38, no. 1, pp. 321-328, 2018.

[25] H.-J. Lee, Z. L. Liang, S. M. Huang et al., "Overexpression of IL-32 is a novel prognostic factor in patients with localized clear cell renal cell carcinoma," Oncology Letters.

[26] J. S. Park, S. Y. Choi, J. H. Lee et al., "Interleukin-32 $\beta$ stimulates migration of MDA-MB-231 and MCF-7cells via the VEGF-STAT3 signaling pathway," Cellular Oncology (Dordrecht), vol. 36, no. 6, pp. 493-503, 2013.

[27] X. Yu, B. Zhou, Z. Zhang et al., "Significant association between IL-32 gene polymorphisms and susceptibility to endometrial cancer in Chinese Han women," Tumour Biology, vol. 36, no. 7, pp. 5265-5272, 2015. 
[28] P. A. T. R. I. C. I. O. GONZALEZ-HORMAZABAL, S. A. N. D. R. A. ROMERO, M. A. H. E. R. MUSLEH et al., "IL-8-251T>A (rs4073) polymorphism is associated with prognosis in gastric cancer patients," Anticancer Research, vol. 38, no. 10, pp. 5703-5708.

[29] P. Gonzalez-Hormazabal, M. Musleh, M. Bustamante et al., "Role of cytokine gene polymorphisms in gastric cancer risk in Chile," Anticancer Research, vol. 34, no. 7, pp. 3523-3530.

[30] A. Untergasser, I. Cutcutache, T. Koressaar et al., "Primer3new capabilities and interfaces," Nucleic Acids Research, vol. 40, no. 15 , p. e115.

[31] X. Sole, E. Guino, J. Valls, R. Iniesta, and V. Moreno, "SNPStats: a web tool for the analysis of association studies," Bioinformatics, vol. 22, no. 15, pp. 1928-1929.

[32] N. Sunaga, H. Imai, K. Shimizu et al., "Oncogenic KRASinduced interleukin-8 overexpression promotes cell growth and migration and contributes to aggressive phenotypes of non-small cell lung cancer," International Journal of Cancer, vol. 130, no. 8, pp. 1733-1744.

[33] N. J. Sullivan, A. K. Sasser, A. E. Axel et al., "Interleukin-6 induces an epithelial-mesenchymal transition phenotype in human breast cancer cells," Oncogene, vol. 28, no. 33, pp. 2940-2947.

[34] Y. Li, L. Wang, L. Pappan, A. Galliher-Beckley, and J. Shi, "IL$1 \beta$ promotes stemness and invasiveness of colon cancer cells through Zeb1 activation," Molecular Cancer, vol. 11, no. 1, p. 87.

[35] J. T. Hong, D. J. Son, C. K. Lee, D. Y. Yoon, D. H. Lee, and M. H. Park, "Interleukin 32, inflammation and cancer," Pharmacology \& therapeutics, vol. 174, pp. 127-137, 2017.

[36] L. A. B. Joosten, B. Heinhuis, M. G. Netea, and C. A. Dinarello, "Novel insights into the biology of interleukin-32," Cellular and Molecular Life Sciences, vol. 70, no. 20, pp. 3883-3892.

[37] B. Heinhuis, M. I. Koenders, W. B. van den Berg, M. G. Netea, C. A. Dinarello, and L. A. B. Joosten, "Interleukin 32 (IL-32) contains a typical $\alpha$-helix bundle structure that resembles focal adhesion targeting region of focal adhesion kinase-1," Journal of Biological Chemistry, vol. 287, no. 8, pp. 5733-5743.

[38] G. Mabilleau and A. Sabokbar, "Interleukin-32 promotes osteoclast differentiation but not osteoclast activation," PLoS ONE, vol. 4, no. 1, p. e4173.

[39] E. Turner-Brannen, K.-Y. G. Choi, R. Arsenault, H. El-Gabalawy, S. Napper, and N. Mookherjee, "Inflammatory cytokines IL-32 and IL-17 have common signaling intermediates despite differential dependence on TNF-receptor 1," The Journal of Immunology, vol. 186, no. 12, pp. 7127-7135.

[40] J. H. Oh, M.-C. Cho, J.-H. Kim et al., "IL-32 $\gamma$ inhibits cancer cell growth through inactivation of NF- $\kappa$ B and STAT3 signals," Oncogene, vol. 30, no. 30, pp. 3345-3359. 